SciDioc

\section{HIV and Suicide: HIV Induces Structural And Functional Abnormalities In White And Grey Matters In The Brain Of Infected People End With Suicide}

Ahed J Khatib ${ }^{1,2^{*}}$

${ }^{1}$ Department of Legal Medicine, Toxicology and Forensic Medicine, Jordan University of Science and Technology, Irbid, Jordan.

${ }^{2}$ International Mariinskaya Academy, Department of Medicine and Critical Care, Department of Philosophy, Academician Secretary of the Department of Sociology, Irbid, Jordan.

Suicide is a prevalent occurrence among those living with HIV/ AIDS. Suicide prevalence estimates, on the other hand, differ amongst studies. In a systematic study, the prevalence of suicidal behavior among HIV/AIDS was calculated. The estimates of the prevalence of suicidal thoughts, attempts, and plans from various studies included in this meta-analysis were pooled using a random-effects model, which was stratified by continent or region and screening instrument. The prevalence of suicide was gathered from 36 studies $(n=32,818)$ from 15 countries. Suicidal ideation, plans, and attempts were estimated to be present in 20.9 percent, 8.1 percent, and 7.5 percentof the population, respectively [2].

HIV is linked to problems with cognition and brain function. Marijuana usage is common among HIV patients, although the effects of marijuana on resting brain function remain unknown. Functional connectivity, or brain activity that is correlated between regions over time, can be used to assess brain function. Disruptions in such connection are becoming more common in neuropsychiatric diseases. During resting state, we looked at the synergistic effects of HIV and marijuana use on functional wholebrain network organization. Our sample consisted of 78 adults with varying HIV and marijuana statuses (19 with co-occurring HIV and marijuana use, $20 \mathrm{HIV-only,} 17$ marijuana-only, and 22 controls). We used eight graph theoretical measures to look at differences in local and long-range brain network organization: transitivity, local efficiency, within-module degree, modularity, global efficiency, strength, betweenness, and participation coefficient. The co-occurring HIV and marijuana use and control groups had similar local and long-range connections. The HIV-only and marijuana-only groups, on the other hand, were both linked to abnormalities in brain network organization. These findings imply that marijuana usage in HIV patients may help to normalize abnormalities in brain network organization. However, more research is needed to identify whether this normalization indicates a favorable or negative effect of marijuana on HIV-infected peo- ple's cognitive functioning [5].

Approximately, 40 million people worldwide are infected with the human immunodeficiency virus (HIV), with over 1.5 million new infections every year [6]. HIV-associated neurocognitive impairment affects $15-50 \%$ of those living with the virus [3]. When the virus infects the central nervous system and triggers a neuroinflammatory cascade, HAND can develop [7]. Even if the condition is under control, the latent virus reservoir can produce ongoing inflammation, which can lead to neuronal damage [4]. HAND causes changes in brain structure and function, including diffuse gray and white matter volume loss, as well as abnormalities in brain function [1].

\section{Our perception}

It is highly possible that HIV impacts on brain are exerted to disrupt logical circuits in the brain as it interferes with white and grey matters in the brain. This may facilitate its existence and escaping from the counteracting actions mediated by immune system.Cognition is also disturbed, and the decision towards may be taken without rational thinking and responsibility. This may be recruited by social impulses and pain intensity. Taken together, our hypothesis states" $\boldsymbol{H I V}$ induces changes in structure and function in the brain of infected persons lead to abnormalities in cognition and committing suicide"

\section{References}

[1]. Chockanathan U, DSouza AM, Abidin AZ, Schifitto G, Wismüller A. Automated diagnosis of HIV-associated neurocognitive disorders using large-scale Granger causality analysis of resting-state functional MRI. Comput Biol Med. 2019 Mar;106:24-30. Pubmed PMID: 30665138

[2]. Pei JH, Pei YX, Ma T, Du YH, Wang XL, Zhong JP, et al. Prevalence of suicidal ideation, suicide attempt, and suicide plan among HIV/AIDS: A systematic review and meta-analysis. J Affect Disord. 2021 Sep 1;292:295-304. Pubmed PMID: 34134028

[3]. McArthur JC, Steiner J, Sacktor N, Nath A. Human immunodeficiency virus-associ-

*Corresponding Author:

Ahed J Khatib,

International Mariinskaya Academy, Department of Medicine and Critical Care, Department of Philosophy, Academician Secretary of the Department of Sociology, Irbid, Jordan. Tel: 00962795905145

E-mail: ajalkhatib@just.edu.jo

Received: January 22, 2022

Published: January 27, 2022

Citation: Ahed J Khatib. HIV and Suicide: HIV Induces Structural And Functional Abnormalities In White And Grey Matters In The Brain Of Infected People End With Suicide. Int J Comput Neural Eng. 2022;8(1e):1-2. doi: http://dx.doi.org/10.19070/2572-7389-220001e

Copyright: Ahed J Khatib ${ }^{\circ} 222$. This is an open-access article distributed under the terms of the Creative Commons Attribution License, which permits unrestricted use, distribution and reproduction in any medium, provided the original author and source are credited. 
ated neurocognitive disorders: Mind the gap. Ann Neurol. 2010 Jun;67(6):699-714. Pubmed PMID: 20517932.

[4]. Saylor D, Dickens AM, Sacktor N, Haughey N, Slusher B, Pletnikov M, et al. HIVassociated neurocognitive disorder - pathogenesis and prospects for treatment. Nat Rev Neurol. 2016 May;12(5):309. Pubmed PMID: 27080521.

[5]. Hall SA, Lalee Z, Bell RP, Towe SL, Meade CS. Synergistic effects of HIV and marijuana use on functional brain network organization. Prog Neuropsychopharmacol Biol Psychiatry. 2021 Jan 10;104:110040. Pubmed PMID: 32687963.
[6]. UNAIDS. Fact Sheet -Latest Global and Regional Statistics on the Status of the AIDS Epidemic. Retrieved from Geneva. 2019.

[7]. Valcour V, Sithinamsuwan P, Letendre S, Ances B. Pathogenesis of HIV in the central nervous system. Current Hiv/aids Reports. 2011 Mar;8(1):54-61. 